\title{
开尔文公式计算蒸气冷凝与液体沸腾温度的误差及其分析
}

\author{
陶智涁, 张树永* \\ 山东大学化学与化工学院, 济南 250100
}

摘要: 讨论了利用开尔文公式计算液体沸腾与蒸气冷凝温度时的误差。指出这些误差是由于没有考虑温度变化对弯 曲液面蒸气压和水平液面蒸气压之比等因素的影响, 以及没有注意开尔文公式对小气泡的适用性导致的。指出了改 善结果的方法, 并进一步明确了开尔文公式的适用范围。

关键词: 开尔文公式; 弯曲液面; 蒸气压; 沸腾; 冷凝

中图分类号: G64; O647

\section{Analysis of the Temperature Deviation for Boiling of Liquid and Condensation of Vapor Using Kelvin Equation}

\author{
Zhibin Tao, Shuyong Zhang * \\ School of Chemistry and Chemical Engineering, Shandong University, Jinan 250100, China.
}

\begin{abstract}
The discrepancies for calculating condensation temperature and boiling temperature for droplet and small bubble using Kelvin equation were discussed. The discrepancies can be ascribed to the neglect of variation of the vapor pressure of curved surface to plain surface with temperature, and the misusing of Kelvin equation for small bubble during boiling. The way to reduce the discrepancy is introduced while the applicability of Kelvin equation is discussed.
\end{abstract}

Key Words: Kelvin equation; Curved surface; Vapor pressure; Boiling; Condensing

\section{1 引言}

开尔文公式(式(1))可以定量描述弯曲液面的饱和蒸气压与其曲率半径的关系 ${ }^{[1]}$ :

$$
\ln \frac{p_{\mathrm{r}}}{p^{*}}=\frac{2 \sigma M}{R T \rho r}
$$

其中, $p_{\mathrm{r}}$ 为弯曲液面的饱和蒸气压, 下标 $\mathrm{r}$ 表示弯曲液面, $p^{*}$ 为水平液面的饱和蒸气压; $\sigma$ 为液 体的表面张力, $M$ 为液体的摩尔质量, $\rho$ 为液体的密度, $T$ 为热力学温度, $R$ 为理想气体常数; $r$ 为 小液滴或小气泡的曲率半径, 对于小液滴, $r>0$; 对于小气泡, $r<0{ }^{[2]}$ 。

关于开尔文公式的讨论很多, 但主要集中于公式的推导, 涉及应用的讨论很少。由于饱和蒸气 压与液体的沸腾和凝聚密切相关, 因此开尔文公式被广泛用于解释液体的过热或者暴沸、蒸气过饱 和与冷凝、毛细凝结等现象 ${ }^{[2-6]}$ 。但进一步分析发现, 虽然现行教材 ${ }^{[2-6]}$ 和参考资料 ${ }^{[7-11]}$ 在使用开尔文 公式解释温度变化的趋势时是合理的, 但进行定量计算时却存在着不可忽视的误差。 


\section{2 开尔文公式计算时的误差}

\section{1 凝聚形成小液滴时的蒸气冷凝温度}

有如下问题: 欲使 $30^{\circ} \mathrm{C}$ 相对湿度 $70 \%$ 的空气中的水蒸气冷凝成半径 $1 \mathrm{~nm}$ 的小液滴, 需要将温 度降低到多少度 ${ }^{[2]}$ ?

经典的计算过程如下: 查阅有关物化手册 ${ }^{[1]}$ 可知, $30{ }^{\circ} \mathrm{C}$ 水的饱和蒸气压为 $4.2470 \mathrm{kPa}$, 表面 张力为 $71.19 \mathrm{mN} \cdot \mathrm{m}^{-1}$, 密度为 $995.61 \mathrm{~kg} \cdot \mathrm{m}^{-3}$ 。取水的摩尔质量为 $18.015 \mathrm{~g} \cdot \mathrm{mol}^{-1}$, 理想气体常数 $8.314 \mathrm{~J} \cdot \mathrm{mol}^{-1} \cdot \mathrm{K}^{-1}$ 。通过开尔文公式计算可得:

$$
\frac{p_{\mathrm{r}}}{p^{*}}=\exp \left(\frac{2 \sigma M}{R T \rho r}\right)=\exp \left(\frac{2 \times 71.19 \times 10^{-3} \times 18.015 \times 10^{-3}}{8.314 \times 303.15 \times 995.61 \times 1 \times 10^{-9}}\right)=2.779
$$

即半径为 $1 \mathrm{~nm}$ 的小液滴的表面饱和蒸气压为水平液面的 2.779 倍, 其蒸气压可达 $11.802 \mathrm{kPa}$ 。 而相对湿度 $70 \%$ 的空气中水的蒸气压只有 $2.973 \mathrm{kPa}$, 因此无法冷凝。欲使空气中的水蒸气冷凝成 $1 \mathrm{~nm}$ 的小液滴, 必须给空气降温, 使 $30^{\circ} \mathrm{C}$ 时的蒸气压达到降温后水的饱和蒸气压的 2.779 倍。由 此求得降温后水的饱和蒸气压应为

$$
p^{*}=4.2470 \times 0.70 \div 2.779=1.0698 \mathrm{kPa}
$$

查阅饱和水蒸气表 ${ }^{[1]}$ 可知, $1.0698 \mathrm{kPa}$ 蒸气压对应的温度为 $7-8{ }^{\circ} \mathrm{C}$ 。

上述计算过程看似合理, 实际却存在问题。这是因为在求解时默认弯曲液面的饱和蒸气压在降 温后不发生变化。而实际上, 由于 $p_{\mathrm{r}} / p^{*}$ 与 $T$ 呈指数反比关系, 当降温到了 $7^{\circ} \mathrm{C}$ 时, 小液滴的饱和蒸 气压与水平面液面的饱和蒸气压比值会变大, 这意味着环境温度须进一步降低。事实上, 不仅温度 $T$ 在变化, 液体的 $\rho$ 和 $p^{*}$ 也随温度而改变。此时需要用迭代法进行求解, 具体求解过程见表 1 。

表 1 利用开尔文公式迭代计算水蒸气冷凝形成半径 $1 \mathbf{n m}$ 小液滴时的温度

\begin{tabular}{ccccc}
\hline 步数 & $T^{\circ} \mathrm{C}$ & $p_{\mathrm{r}} / p^{*}$ & $p^{* *} / \mathrm{kPa}$ & $T^{v} /{ }^{\circ} \mathrm{C}$ \\
\hline 1 & 30.00 & 2.779 & 1.0698 & 7.956 \\
2 & 7.956 & 3.155 & 0.9423 & 6.107 \\
3 & 6.107 & 3.192 & 0.9314 & 5.939 \\
4 & 5.939 & 3.195 & 0.9305 & 5.925 \\
5 & 5.925 & 3.196 & 0.9302 & 5.920 \\
6 & 5.920 & 3.196 & 0.9302 & 5.920 \\
\hline
\end{tabular}

$T:$ 环境温度; $p^{* *}$ : 对应的饱和蒸汽压; $T^{\prime}$ : 对应温度

计算过程中采用的物性数据见表 2:

表 2 计算过程中使用的物性数据 ${ }^{[1]}$

\begin{tabular}{cccc}
\hline$T /{ }^{\circ} \mathrm{C}$ & $p^{*} / \mathrm{kPa}$ & $\sigma /\left(\mathrm{mN} \cdot \mathrm{m}^{-1}\right)$ & $\rho /\left(\mathrm{kg} \cdot \mathrm{m}^{-3}\right)$ \\
\hline 30.00 & 4.2470 & 71.19 & 995.61 \\
7.956 & 1.0698 & 74.52 & 999.81 \\
6.107 & 0.9423 & 74.78 & 999.90 \\
5.939 & 0.9314 & 74.81 & 999.91 \\
5.925 & 0.9305 & 74.81 & 999.91 \\
5.920 & 0.9302 & 74.81 & 999.91 \\
\hline
\end{tabular}

$p^{*}$ : 水的饱和蒸汽压; $\sigma$ : 水的表面张力; $\rho$ : 水的密度 
表 1 显示, 当迭代到第 6 步时温度不再变化, 稳定在 $5.920{ }^{\circ} \mathrm{C}$ 。该结果与采用一步计算所得结 果 $7.956{ }^{\circ} \mathrm{C}$ 相比, 降低超过 $2{ }^{\circ} \mathrm{C}$, 相对误差达 $26 \%$ 。

由于指数函数对于微小变化的敏感性, 虽然 $p_{\mathrm{r}} / p^{*}$ 比值随 $T$ 的改变量并不大, 但仍对最终的温度 造成了较大的影响。

\section{2 有小气泡存在时的液体沸腾温度}

在讨论液体过热和暴沸时, 很多教材 ${ }^{[2-6]}$ 都利用开尔文公式进行解释。

相关计算有 2 种思路: 一是对于在小气泡内气化的水而言, 由于附加压力的存在导致其蒸气压 下降, 需要通过提高液体的温度提高水的蒸气压, 使之达到 1 个大气压, 水才能沸腾; 二是考虑附 加压力导致小气泡中的气压升高, 相当于液体气化需要反抗的外压增加。为了使液体沸腾也必须给 液体进一步升温。从理论上说, 这两种思路应该得到相同的结论。但在教材的例题和课后习题中, 却往往不使用开尔文公式, 而是使用拉普拉斯公式计算附加压力后, 再利用克劳修斯-克拉佩龙方程 计算液体的沸腾温度 ${ }^{[2,4,8]}$ 。本文以教材 ${ }^{[2]} 291$ 页习题 4 为例进行说明: 如果水中仅含半径为 $1 \mu \mathrm{m}$ 的 空气泡, 试求这样的水开始沸腾的温度是多少?

为了简化计算, 我们忽略了液体静压力对小气泡的影响。查阅手册 ${ }^{[1]}$, 取 $100^{\circ} \mathrm{C}$ 下水的表面张 力为 $58.91 \mathrm{mN} \cdot \mathrm{m}^{-1}$, 密度为 $958.35 \mathrm{~kg} \cdot \mathrm{m}^{-3}$ 。根据开尔文公式, 则蒸气压的比值应为:

$$
\frac{p_{\mathrm{r}}}{p^{*}}=\exp \left(\frac{2 \sigma M}{R T \rho r}\right)=\exp \left(\frac{2 \times 58.91 \times 10^{-3} \times 18.015 \times 10^{-3}}{8.314 \times 373.15 \times 958.35 \times-1 \times 10^{-6}}\right)=0.999
$$

迭代计算如表 3:

表 3 开尔文公式迭代计算水沸腾温度

\begin{tabular}{ccccc}
\hline 步数 & $T /{ }^{\circ} \mathrm{C}$ & $p_{\mathrm{r}} / p^{*}$ & $p^{* *} / \mathrm{kPa}$ & $T / /{ }^{\circ} \mathrm{C}$ \\
\hline 1 & 100.00 & 0.999 & 101.431 & 100.0034 \\
2 & 100.0034 & 0.999 & 101.431 & 100.0034 \\
\hline
\end{tabular}

表 3 结果显示, 当气泡半径为 $1 \mu \mathrm{m}$ 时, 弯曲液面液体的蒸气压与水平面液体的相差极小, 经过 2 次迭代即可达到稳定, 温度为 $100.0034{ }^{\circ} \mathrm{C}$ 。这表明采用开尔文公式预测的蒸气压变化幅度很小, 对沸点的影响几乎可以忽略。

接下来通过附加压力导致外压增加的方法 ${ }^{[2,4]}$ 求解。首先计算小气泡的附加压力:

$$
\Delta p=\frac{2 \sigma}{r}=-\frac{2 \times 58.91 \times 10^{-3}}{10^{-6}}=-117.82 \mathrm{kPa}
$$

负号表示压力朝向气相。取 $p_{1}=101.325 \mathrm{kPa}$, 则小气泡的内部压力为:

$$
p_{2}=p_{1}-\Delta p=101.33+117.82=219.15 \mathrm{kPa}
$$

根据克劳修斯-克拉佩龙方程:

$$
\ln \frac{p_{2}}{p_{1}}=-\frac{\Delta_{\text {vap }} H_{\mathrm{m}}}{R}\left(\frac{1}{T_{2}}-\frac{1}{T_{1}}\right)
$$

取水的摩尔蒸发焓 $\Delta_{\mathrm{vap}} H_{\mathrm{m}}=40.649 \mathrm{~kJ} \cdot \mathrm{mol}^{-1}, T_{1}=373.15 \mathrm{~K}{ }^{[1]}$, 则有

$$
\begin{aligned}
& \frac{1}{T_{1}}-\frac{1}{T_{2}}=\frac{R \times \ln \left(p_{2} / p_{1}\right)}{\Delta_{\text {vap }} H_{\mathrm{m}}}=\frac{8.314 \times \ln \left(\frac{219.15}{101.33}\right)}{40.649 \times 10^{3}}=1.578 \times 10^{-4} \mathrm{~K}^{-1} \\
& T_{2}=396.50 \mathrm{~K}=123.35{ }^{\circ} \mathrm{C}
\end{aligned}
$$

计算表明, 如果水中只存在半径为 $1 \mu \mathrm{m}$ 的小气泡, 水的沸腾温度为 $123.35^{\circ} \mathrm{C}{ }^{[2]}$ 。此温度与开 尔文公式求解的结果 $\left(100.0034{ }^{\circ} \mathrm{C}\right)$ 有较大出入。 
从上面的计算可以看出, 对于液体的沸腾而言, 由弯曲液面导致蒸气压变化所预测的沸点, 远 远小于附加压力导致外压变化预测的沸点。这说明在讨论过热液体沸腾时, 即便是定性解释也应从 附加压力的角度考虑, 而不应使用开尔文公式。

\section{3 开尔文公式误差产生的原因分析}

通过之前的叙述可以发现, 开尔文公式在具体应用时会存在不可忽视的误差。这种误差可分为 两类: 忽略温度变化对 $p_{\mathrm{r}} / p^{*}$ 比值影响所导致的误差(误差-1), 以及忽略开尔文公式适用性导致的误 差(误差-2)。其中, 误差-1 已在 2.1 部分做了详细讨论, 此处不再赘述。误差-2 则是导致 2.2 问题的 原因。

对处于平衡状态的气/液两相, 应同时满足相平衡与力平衡两个条件。相平衡保证了两相之间不 因化学势的不同而发生物质的转移; 力平衡保证两相之间不会因为受力不同而发生相对位移。

对于一个由气相 $(\mathrm{G})$ 、液相 $(\mathrm{L})$ 和界面相 $(\sigma)$ 组成的系统, 在恒温条件下, 这两个条件可以描述 为 ${ }^{[7]}$ :

$$
\begin{aligned}
& p_{\mathrm{L}}=p_{\mathrm{G}}+\sigma \frac{\mathrm{d} A_{\sigma}}{\mathrm{d} V_{\mathrm{L}}} \\
& \mu_{\mathrm{L}}=\mu_{\mathrm{G}}
\end{aligned}
$$

其中 $p_{\mathrm{L}}$ 和 $p_{\mathrm{G}}$ 分别是液相和气相的压力, 不是蒸气压。对于水平液面, 界面相的面积 $A_{\sigma}$ 是容器 形状的函数, 与液体体积 $V_{\mathrm{L}}$ 无明确关系。因此

$$
\begin{aligned}
& \frac{\mathrm{d} A_{\sigma}}{\mathrm{d} V_{\mathrm{L}}}=0 \\
& p_{\mathrm{L}}=p_{\mathrm{G}}=p^{*}
\end{aligned}
$$

基于力平衡条件(3)，假设液滴呈球形，带入球体的体积和表面积公式:

$$
\begin{aligned}
& V_{\mathrm{L}}=\frac{4 \pi r^{3}}{3} \\
& A_{\sigma}=4 \pi r^{2}
\end{aligned}
$$

即可得到拉普拉斯公式:

$$
p_{\mathrm{L}}-p_{\mathrm{G}}=\Delta p=\frac{2 \sigma}{r}
$$

即液滴所受的压力与外界气相压力之间的压力差为附加压力, 两者处于力平衡。对于相平衡条 件(4), 考虑到力平衡条件已满足, 两相间无相对运动, 表面相的面积保持不变。则对于小液滴, 代 入气液两相化学势公式:

$$
\begin{aligned}
& \mathrm{d} \mu_{\mathrm{g}}=R T \mathrm{~d} \ln p_{\mathrm{g}} \\
& \mathrm{d} \mu_{1}=R T \mathrm{~d} \ln p_{\mathrm{r}}=V_{\mathrm{m}} \mathrm{d} p_{\mathrm{L}}
\end{aligned}
$$

其中, $V_{\mathrm{m}}$ 表示液体的摩尔体积, 下标 $\mathrm{m}$ 表示摩尔。取 $p_{\mathrm{r}}$ 为弯曲液面的蒸气压, 再取 $p^{*}$ 为水平 液面的蒸气压。从水平液面向小液滴积分, 代入公式(6)和(9), 即水平液面时两相压力相等, 小液滴 的 $p_{\mathrm{L}}$ 多一个附加压力:

$$
\int_{p^{*}}^{p_{\mathrm{r}}} R T \mathrm{~d} \ln p=\int_{p_{\mathrm{G}}}^{p_{\mathrm{G}}+\frac{2 \sigma}{r}} V_{\mathrm{m}} \mathrm{d} p_{\mathrm{L}}
$$

如果认为 $V_{\mathrm{m}}$ 保持不变, 积分可得:

$$
R T \ln \frac{p_{\mathrm{r}}}{p^{*}}=\frac{M}{\rho}\left(\frac{2 \sigma}{r}\right)
$$

此即开尔文公式(1)。

对于分散在气体中的小液滴, 由于液体分子间存在斥力, 可以抵抗向内的附加压力, 液滴不发 
生形变, 满足力平衡条件。然而, 由于表面分子的能量高, 导致弯曲液面的饱和蒸气压变大, 化学 势不平衡, 液滴趋向于蒸发。尤其是在液滴形成的初期 $r$ 极小, 这种情况更为明显。所以, 小液滴 难以形成, 此即为蒸汽的过饱和现象。

而对于分散在液体中的小气泡, 其所受压力 $\left(p_{\mathrm{ex}}\right)$ 包括大气压力 $\left(p_{\mathrm{g}}\right)+$ 液体静压力 $\left(p_{1}\right)+$ 附加压力 $(\Delta p)$; 小气泡反抗外压的压力 $\left(p_{\text {in }}\right)$ 只有饱和蒸汽的压力 $\left(p_{\text {vap }}\right)$ 。在小气泡形成初期, 附加压力极大, 外 压明显大于内压, 因此小气泡难以形成。解决这一问题的方法之一是升温, 直到饱和蒸汽压力能够 抵御外压 $\left(p_{\mathrm{ex}}\right)$, 这就导致液体的过热现象; 二是通过加入沸石等方法在液体内部引入具有较大尺寸 的空气泡, 使气泡内压变为大气压 $\left(p_{\mathrm{g}}\right)+$ 液体蒸气压 $\left(p_{\mathrm{vap}}\right)$, 一方面抵消外界大气压, 另一方面增大小 气泡的初期半径以减小附加压力 ${ }^{[9]}$ 。

实际上, 在液体气化、小气泡长大的过程中, 液态水转化为气态水, 体积产生巨大变化, 导致 附加压力和体积均发生改变, 式(11)不再成立。因此, 对于液体气化形成的小气泡, 是无法导出开尔 文公式的。虽然有其他方法 ${ }^{[2-8,12-17]}$ 推导开尔文公式, 但是推导过程也都只是从小液滴出发, 得出公 式后再推广至小气泡, 而这种推广的前提实际上是不存在的。

\section{4 结语}

通过两个实例分析了使用开尔文公式计算蒸气冷凝和液体沸腾温度过程中所产生的误差，分析 了导致误差的原因, 提出了消除误差的方法。进一步讨论了开尔文公式对小气泡的适用性, 辨析了 求解液体沸腾温度的正确方法。

\section{参 考 文 献}

[1] Haynes, W. M. CRC Handbook of Chemistry and Physics, 97th ed.; CRC Press: Boca Raton, 2017; pp. 6-1.

[2] 印永嘉, 奚正楷, 张树永. 物理化学简明教程. 第 4 版. 北京: 高等教育出版社, 2007: 286-292.

[3] 重庆大学物理化学教研室. 物理化学. 重庆: 重庆大学出版社, 2008: 206-210.

[4] 傅献彩, 沈文霞, 姚天扬, 侯文华. 物理化学. 第 5 版. 北京: 高等教育出版社, 2007: 323-324.

[5] 李元高. 物理化学. 上海: 复旦大学出版社, 2013: 197-202.

[6] 周鲁. 物理化学教程. 第 4 版. 北京: 科学出版社, 2017: 211-220.

[7] 刘国杰, 黑恩成. 物理化学导读. 北京: 科学出版社, 2008: 294-301.

[8] 刘国杰, 黑恩成. 物理化学释疑. 北京: 科学出版社, 2010: 133-138.

[9] 孙朗克. 辽宁教育学院学报, 1996, No. 5, 67.

[10] 刘幸平. 物理化学习题集. 北京: 中国中医药出版社, 2005: 98-107.

[11] 李支敏, 王保怀, 高盘良. 物理化学解题思路和方法. 北京: 北京大学出版社, 2002: 340-342.

[12] 梁嘉树, 郭东升. 大学化学, 2019, 34 (2), 55.

[13] 邱如斌, 邹开煌. 福建教育学院学报, 2003, No. 4, 70.

[14] 李献文. 福建教育学院学报, 2004, No. 1, 44.

[15] 马淑清, 王鉴, 桑希勤, 李丽丽, 吴松. 化学教育, 2016, 37 (18), 75.

[16] 梁山, 崔玉红, 郭艳玲. 大学化学, 2013, 28(4), 67.

[17] 王基镕. 大学物理, 2007, 26 (9), 4. 